Meta

Journal des traducteurs

Translators' Journal

\title{
Figement et dénomination
}

\section{Salah Mejri}

Volume 45, numéro 4, décembre 2000

URI : https://id.erudit.org/iderudit/003611ar

DOI : https://doi.org/10.7202/003611ar

Aller au sommaire du numéro

\section{Éditeur(s)}

Les Presses de l'Université de Montréal

ISSN

0026-0452 (imprimé)

1492-1421 (numérique)

Découvrir la revue

Citer cet article

Mejri, S. (2000). Figement et dénomination. Meta, 45(4), 609-621.

https://doi.org/10.7202/003611ar

\section{Résumé de l'article}

Les séquences figées, grâce à leurs caractéristiques linguistiques (oblicité des dénominations, stratification de leurs structures et polylexicalité de leurs signifiants), offrent l'occasion d'étudier sous un angle nouveau certains concepts fondamentaux de la linguistique moderne comme la référence, la conceptualisation, l'arbitraire du signe, etc., et ce, en rapport étroit avec l'acte de dénomination, ce qui permet de mieux articuler et de hiérarchiser les opérations de référenciation, de conceptualisation et de formation de l'unité linguistique dans la genèse du signe.
Ce document est protégé par la loi sur le droit d'auteur. L’utilisation des services d’Érudit (y compris la reproduction) est assujettie à sa politique d'utilisation que vous pouvez consulter en ligne.

https://apropos.erudit.org/fr/usagers/politique-dutilisation/ 


\title{
Figement et dénomination
}

\author{
SALAH MEJRI \\ Université de Tunis I, Tunis, Tunisie
}

\begin{abstract}
RÉSUMÉ
Les séquences figées, grâce à leurs caractéristiques linguistiques (oblicité des dénominations, stratification de leurs structures et polylexicalité de leurs signifiants), offrent l'occasion d'étudier sous un angle nouveau certains concepts fondamentaux de la linguistique moderne comme la référence, la conceptualisation, l'arbitraire du signe, etc., et ce, en rapport étroit avec l'acte de dénomination, ce qui permet de mieux articuler et de hiérarchiser les opérations de référenciation, de conceptualisation et de formation de l'unité linguistique dans la genèse du signe.
\end{abstract}

\section{ABSTRACT}

Thanks to their linguistic characteristics (obliqueness of denominations, stratification of their structure and polylexicality of their signifier), fixed phrases offer the opportunity to study from a new angle some basic concepts of modern linguistics such as reference, conceptualization, the arbitrariness of the sign, etc., and this is in close connection with the act of denomination, which makes it possible to organize and structure the operations of referentiation, conceptualization and the formation of the linguistic sign in the geneses of the sign.

\section{MOTS-CLÉS/KEYWORDS}

dénomination, figement, conceptualisation, référence, polylexicalité

1. Ce titre est problématique à plusieurs égards: d'abord, il associe deux entités de nature différente, le figement étant un processus linguistique alors que la dénomination renvoie à l'acte par lequel on attribue un nom aux entités de l'univers, même si le terme est employé en même temps métonymiquement pour référer au produit de cette opération; il cultive en outre un flou dans le rapport qu'il y a à établir entre les deux termes du titre.

L'objectif de cette formulation est de limiter la réflexion à un niveau méthodologique général parce que les liens entre les deux sphères de réflexion sont loin d'être défrichés, et ce, en raison de leur marginalisation dans la recherche linguistique.

1.1. Le figement, qui commence à peine à faire partie des préoccupations majeures des linguistes, n'a pas pour autant bénéficié jusque-là d'une définition relativement reconnue par tout le monde. Il demeure une notion obscure rappelant d'autres termes qui couvrent plus ou moins le même contenu comme lexies complexes, phraséologies, syntagmes lexicalisés, expressions idiomatiques, locutions, etc. (pour une liste plus détaillée, cf. Kocourek 1982: 117). Toutefois, une lecture attentive des études récentes consacrées à ce phénomène (Makkaï 1972; Gréciano 1983; G. Gross 1996; M. Gross 1988; Mejri 1994, 1997, 1998) permet de dégager ces éléments de définition qui, sans rendre compte de l'extrême complexité de ce phénomène, nous aideraient à avancer sûrement dans nos investigations : 
(1) Le figement est un processus linguistique inhérent aux langues naturelles par lequel des séquences linguistiques, initialement employées comme séquences discursives libres, se trouvent, pour des raisons diverses, partiellement ou entièrement solidifiées; elles sont ainsi versées dans l'une des catégories linguistiques dans le cadre de laquelle les constituants perdent leur autonomie individuelle pour participer à la configuration de la nouvelle unité polylexicale ainsi constituée.

Cette définition laisse dans l'ombre plusieurs aspects, dont notamment les raisons qui sont à l'origine de ce phénomène, mais cela ne nous empêche pas, à ce point de l'analyse, d'avoir une idée relativement précise du phénomène puisqu'il nous est possible de dégager de cette définition des éléments théoriques qui nous seront très utiles dans la suite de notre exposé. Retenons la notion de processus qui implique l'idée de la succession dans le temps d'un ensemble de phénomènes ou d'opérations conduisant à un résultat (Grand Larousse de la langue 1987), celle du caractère inhérent aux langues naturelles qui implique que toute langue employée conduit nécessairement au déclenchement d'un tel processus en tant que facteur d'existence - d'aucuns diraient de survie - du système linguistique, et finalement celle de systémicité, puisque les séquences figées (SF) sont le produit du fonctionnement général du système et, par conséquent, le reflet et l'implication de son économie générale.

Si nous n'avons pas évoqué jusque-là la fonction référentielle de ces SF, c'est parce que la dénomination représente le second terme de notre intitulé, terme non moins problématique.

1.2. Tout comme le figement, la dénomination n'a pas bénéficié d'un intérêt particulier de la part des linguistes - ce terme ne figure même pas dans le dictionnaire de Dubois - parce que, nous semble-t-il, cette notion a une dimension philosophique certaine, ce qui n'a pas favorisé la réflexion linguistique sur la question. Cette dimension est d'autant plus philosophique que la réflexion sur la dénomination entraîne facilement dans des domaines aussi complexes que le rapport avec le monde, c'est-àdire tout ce à quoi renvoient les unités linguistiques, qu'il soit réel ou non, qu'il relève des choses, des êtres, des idées, des procès, des façons d'être; bref, tout ce qui est nommable. Très rapidement, la réflexion sur la dénomination touche à des préoccupations à caractère ontologique.

Bien qu'il ne soit pas interdit aux linguistes de s'occuper de problèmes ontologiques - qu'y a-t-il de plus ontologique que les langues? - , il semble que des préoccupations épistémologiques relatives à la délimitation de l'objet de la discipline l'aient emporté pour une très longue période, laissant ainsi cette question, bien que strictement linguistique comme son nom l'indique, hors des champs d'investigation des linguistes. Même si on lui a consacré quelques travaux (cf. Langages 1984; Boisson et Thoiron 1997), on l'a fait sous des angles bien particuliers, privilégiant ainsi dans chaque cas d'autres préoccupations dans lesquelles la dénomination est nécessairement impliquée. Dans le numéro de Langages, la question centrale demeure la dimension pathologique, plus précisément l'impact de l'aphasie sur la dénomination et les problèmes psychologiques rattachés à la dénomination. Dans l'ouvrage de l'équipe du CRTT de Lyon, c'est par le biais de la terminologie que les auteurs ont abordé cette question. Par ailleurs, il est à signaler que les bibliographies figurant dans ces deux recueils de textes mentionnent très rarement des écrits portant exclusivement sur la dénomination. Le numéro spécial de Meta sur la dénomination dirigé 
par Philippe Thoiron (41-4, déc. 1996) n'échappe pas à la règle. Bien que les descriptions présentées portent sur des aspects linguistiques divers (cf., par exemple, Kleiber (1996: 567-589) sur les noms propres et les noms communs), elles sont loin d'aboutir à une définition générale de la dénomination pouvant servir de repère à une analyse de nature strictement linguistique, c'est-à-dire privilégiant le linguistique comme aspect dominant dans l'acte de dénommer.

À mon avis, c'est à Kleiber que nous devons les études les plus théoriques sur la question, ce qui ne l'empêche pas de préciser qu' «en linguistique, la dénomination est un concept aux contours mal délimités dont l'extension varie considérablement selon les théories et les auteurs» (1984: 77).

Ce qu'on peut affirmer, c'est qu'actuellement la dénomination commence à avoir droit de cité en tant que question linguistique non seulement digne d'intérêt mais dont la valeur heuristique est certaine. La réflexion menée dans ce domaine a permis à la linguistique de revendiquer certains domaines jusque-là considérés comme non linguistiques. Nous rappelons à titre d'exemple tout ce qui intéresse cette zone floue qui intéresse tous les processus cognitifs producteurs de dénominations, les opérations intellectuelles par lesquelles on passe de l'univers au signe linguistique, et ce saut qualitatif par lequel l'esprit transforme les données massives et fluctuantes du vécu en entités discontinues maîtrisables par la seule présence du signe linguistique.

Ainsi faudrait-il retenir des questions aussi intéressantes que:

- le rapport dénomination/signe linguistique,

- le statut du référent dans cette opération,

- la place qu'occupe le concept par rapport au référent et au signifié,

- les implications que telles précisions peuvent avoir sur ce qu'il est convenu d'appeler l'arbitraire du signe.

S'agissant du premier point, il y a lieu de remarquer qu'un grand flou entoure les définitions les plus courantes de la dénomination, comme «le fait de donner un nom à quelque chose» (Le grand dictionnaire des lettres [GDL], vol. 8) ou «la relation qui unit une expression linguistique à une entité extra-linguistique» (Kleiber 1984: 77), et ce, en raison de l'imprécision des termes définitoires employés tels que «nom» et "quelque chose», ou du caractère très générique de formules telles qu' "expression linguistique». Dès qu'on cherche à être plus précis, les difficultés apparaissent.

S'agit-il d'une opération orientée prenant son point de départ vers le signe ou du signe linguistique? L'expression linguistique est-elle un signe linguistique dans le sens saussurien du terme ou un simple signifiant? S'il s'agit du signe linguistique avec ses deux faces, signifiant et signifié, quel rapport doit exister entre le signifié et le référent? Ce rapport est-il médiat ou immédiat?

D'où la seconde interrogation relative au statut du référent dans l'acte de dénommer à laquelle il faudrait rattacher des questions aussi simples que la définition du référent: doit-il être appréhendé dans sa matérialité ou dans son abstraction en tant qu'entité construite par l'esprit lors de son appréhension du monde? Doit-il être conçu dans sa singularité ou dans sa pluralité?

Autant de questions qui nous conduisent à la conceptualisation en tant qu'étape nécessaire dans la construction du langage, tout langage, ce qui complique davantage la situation parce qu'en plus du référent on se trouve en présence de cet autre élément 
constitutif de la dénomination qu'est le concept. Il y aurait ainsi au moins quatre éléments dont il faut préciser et la nature et le statut dans la dénomination: le référent, le concept, le signifié et le signifiant. Se présentent-ils dans un ordre hiérarchisé ou interviennent-ils d'une manière concomitante?

2. Nous pensons que l'étude des séquences figées dénominatives peut aider à éclairer certains aspects de ces difficultés. Cottez (1987: 75) précise à propos de la dénomination que:

(2) Les êtres et les objets du monde où vit un peuple ne sont pas appelés n'importe comment dans la langue de ce peuple: il y a des habitudes de nommer [...]. Et ces habitudes sont liées dans le lexique. Les habitudes de nommer ne se reconnaissent pas dans les signes arbitraires (hérités ou importés), mais dans les dénominations motivées, formées sur la base des signes arbitraires sous l'effet de pratiques onomasiologiques peu à peu enracinées, qui résistent même aux changements morphologiques.

De son côté, Lobatchev, en évoquant les séquences figées, cite cette «perle» : «Il faut retrousser ses manches... pour donner un coup de pouce», en ajoutant qu'à l'interprétation globale échappe une nuance «qui reste enfouie sous une enveloppe conventionnelle du tour phraséologique»; et Lobatchev de conclure (1987: 124):

(3) «On a beau parler des idiotismes phraséologiques pour proclamer leur arbitraire, on n'arrive pas à oublier le sens lexical de leurs composants.»

Partant de ces réflexions, nous voudrions montrer dans ce qui suit que les SF représentent des signes linguistiques qui offrent le moyen de mener une réflexion sur plusieurs aspects de la dénomination dont les unités lexicales simples rendent compte très difficilement. Nous montrerons d'abord comment les SF permettent de préciser clairement les différentes étapes impliquées dans la dénomination et comment leur structuration liguistique héritée de leur genèse conditionne leur fonctionnement dans le discours.

2.1. En voulant distinguer l'interprétation métalinguistique (relative aux noms communs) de l'interprétation non linguistique (relative aux noms propres), Kleiber oppose les SN élémentaires (unités simples) aux SN complexes (unités polylexicales) en montrant à partir des exemples (4) et (5) que seuls les derniers sont susceptibles des deux interprétations alors que les premiers n'admettent que l'interprétation non métalinguistique:
(4) - Comment s'appelle la chèvre?
- Quel est le nom de la chèvre?
( $\mathrm{SN}$ générique) / ( $\mathrm{SN}$ spécifique)
(5) - Comment s'appelle la gazelle autrichienne? (SN générique) / (SN spécifique) - Quel est le nom de la gazelle autrichienne?

Lorsque le SN est générique, l'interprétation en 4 s'avère une tautologie (1984: 92).

Cet argument employé par Kleiber pour marquer la différence entre le nom propre et le nom commun peut nous servir de point de départ pour montrer que le caractère polylexical des SF (= SN complexes), qui ajoute en fait une articulation supplémentaire à la chaîne syntagmatique (cf. Mejri 1998), trahit une dualité struc- 
turale qui fait qu'on dit quelque chose pour signifier autre chose. Dans l'exemple suivant:

(6) $[\mathrm{P}]$ endant que votre comtesse riait, dansait, faisait ses singeries, elle était dans ses petits souliers, comme on dit, en pensant à ses lettres de change protestées, ou à celles de son amant. (Honoré de Balzac, Le Père Goriot, cité par Rey et Chantreau 1989)

«être dans ses petits souliers», qui signifie 'être mal à l'aise', impose le commentaire métalinguistique qui justifie en quelque sorte son emploi.

Pourquoi de tels commentaires? Parce que c'est tout simplement une façon de dire qui marque un décalage entre le sens compositionnel et le sens global. Dans cet exemple, il n'est pas question de «souliers», si petits soient-ils. En fait, l'articulation supplémentaire évoquée, qui s'interpose entre les unités de la première articulation, ici la séquence globale, et celles de la deuxième articulation, c'est-à-dire les phonèmes, est formée d'unités qui ne sont pas étanches au sens puisqu'elles sont des unités de première articulation si elles sont employées hors du cadre de la SF, leur emploi dans les SF repose nécessairement sur un dédoublement qui fait que ces unités participent à la dénomination polylexicale et conservent, comme l'a si bien vu Lobatchev, des traces de leurs emplois en tant qu'unités simples sans que cela verse dans la polysémie: dans (6), il serait inapproprié de parler de polysémie de "petits» ou de «souliers».

2.2. C'est ce dédoublement qui nous offre le moyen de remonter le parcours fait par la SF depuis la référence jusqu'à ce signifiant global qui a servi de cadre à la synthèse sémantique à l'origine de son signifié non compositionnel. Si on considère la relation dénominative comme une «relation référentielle codée qui unit un lexème à son référent» (Mortureux 1995: 448) ou comme «l'établissement d'une règle de fixation référentielle qui permet l'utilisation ultérieure du nom pour l'objet dénommé» (Kleiber 1984: 80), on ne peut pas s'empêcher de relever la rupture ou le décrochage référentiel opérés au niveau des unités simples constitutives de la SF, ce qui signifie que ces unités sont employéss indépendamment des références qu'elles dénomment initialement même si cette suspension n'est ni totale ni durable (cf. Mejri 1998 pour la continuité référentielle dans les séquences figées).

En fait, l'accrochage référentiel de la SF se fait sur la base d'entités référentielles déjà existantes qui s'éclipsent au profit de la référence nouvelle tout en gardant dans leur structure stratifiée les relations référentielles de base, ce qui permet les remotivations de toutes sortes:

(7) La conjonction de ces trois difficultés nous a conduit lors du bilan de l'année dernière à abandonner un des points centraux de notre analyse de 1981, l'hypothèse que les noms propres étaient des prédicats de dénomination. Nous n'avons pas pour autant jeté le bébé dénominatif avec l'eau de ce bain prédicatif, puisque nous avons maintenu l'hypothèse d'un sens de dénomination pour le nom propre. (Kleiber 1996: 573)

Ce qui donnerait des emplois où la réactivation des rapports référentiels initiaux crée une sur-référenciation, fruit de la superposition qu'on pourrait représenter comme suit: 


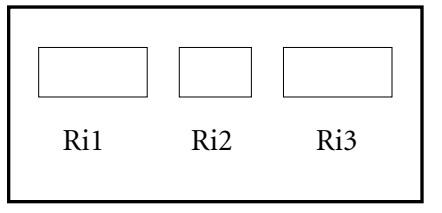

$\operatorname{Rg}$

Le décrochage référentiel des unités constitutives dans la SF.

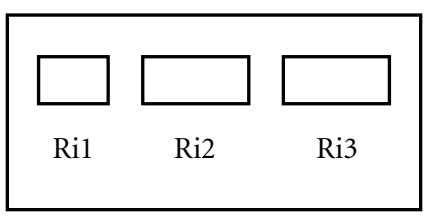

$\operatorname{Rg}$

La réactivation des références de base dans les emplois remotivés (cf. l'exemple cité par Lobatchev).

Nous nous sommes interrogés au début sur la nature du référent, s'il est pluriel ou singulier; nous croyons que nous pouvons maintenant préciser:

- que le référent est ce qui, dans le monde, justifie et fonde l'existence du signe, c'est-àdire la contrepartie du contrat sémiotique par lequel un symbole n'assure sa fonction représentative que s'il renvoie à une entité dont il traduit l'existence par sa simple réalisation (cf. la "présupposition d'existence d'univers», Martin 1992: 49). C'est la nature de cette relation qui permet dans le langage la substitution du symbole au référent et qui autorise l'existence du symbole indépendamment du référent (cf. aussi les emplois autonymiques où le signe devient son propre référent).

- $\quad$ que le référent ne peut être qu'une entité antinomique puisqu'il véhicule en lui «une singularité plurielle» ou une "pluralité singulière». Il ne s'agit pas là d'un jeu de mot. Nous croyons que c'est cette hétérogénéité antinomique qui fournit au fonctionnement des unités lexicales l'homogénéité nécessaire à toute unité (cf. les notions d'extension et de compréhension).

Cette précision nous permet de résoudre plusieurs problèmes à la fois :

- le fait que la même unité lexicale simple peut renvoyer à plus d'un référent, par exemple: mère: personne, moule en plâtre ou membrane gélatineuse formée à la surface d'un liquide alcoolique (mère de vinaigre);

- le fait que des références soient superposées dans le cadre de la même unité lexicale sans qu'il y ait dans le monde de rapports qui soient de nature à les intégrer dans une structure commune (cf. le rapport entre le référent de "bébé» avec le référent de la séquence «jeter le bébé avec l'eau du bain » qu'est l'attitude qu'on pourrait avoir dans certaines situations);

- le fait que l'extension de l'unité lexicale, de par sa nature plurielle, prête à la compréhension des éléments nécessairement réduits mais suffisamment représentatifs pour que le contrat sémiotique qui lie le signe au référent reste fonctionnel même si un référent particulier ne répond pas à certains éléments de la compréhension (cf. l'exemple tant discuté d'«oiseau»).

2.3. Dès que l'accrochage référentiel de la SF est établi, s'amorce l'opération de conceptualisation qui prend son point de départ dans cette singularité déjà évoquée. Nous avons analysé cette opération ailleurs (Mejri 1998); rappelons seulement que c'est une étape cruciale parce qu'elle détermine la suite de la construction du signe linguistique. Claude Vandeloise (1991: 73), par exemple, rappelle que «l'existence d'une pensée prélinguistique ne peut [...] être exclue a priori, et on croit même, en linguistique cognitive, que la conceptualisation prélinguistique du monde est loin d'être amorphe». 
Puisqu'il s'agit d'une opération de grande abstraction par laquelle l'hétérogénéité de l'univers est ramenée à une homogénéité structurelle et la complexité de l'univers réduite à des structures relativement simples, le concept apparaît comme l'unique médiation possible entre le monde et le langage. Précisons cependant que tout concept n'est pas nécessairement dénommé; il peut faire l'objet d'autres formes d'expressions, icôniques par exemple. Il arrive même qu'il fasse l'objet de plusieurs désignations contingentes avant de se fixer dans un signe linguistique (cf. le parcours des néologismes, Mejri 1995).

Cette étape offre à la langue la matière conceptuelle nécessaire à la formation du signe linguistique, laquelle obéit aux contraintes de nature strictement linguistique (les catégories grammaticales, la configuration simple ou complexe du signifiant, les paradigmes lexicaux disponibles, etc.).

En s'intéressant à cet aspect, Henri Béjoint et Philippe Thoiron (1996: 512-524), avec la collaboration de Pierre Arnaud et Claude Pierre Boisson, ont pu dégager à partir de l'étude des dénominations de «la règle à calculer» dans plusieurs langues un «archi-concept» à la représentation synthétique suivante:

\section{(9) /RÈGLE/, /CALCULER/, /COULISSER/, /LOGARITHMIQUE/, /POUR INGÉNIEURS/}

dont la réalisation dénominative ne sélectionne pas nécessairement dans chaque langue les mêmes traits conceptuels. Si on compare traits conceptuels et éléments de nomination, on constate que le français dénomme cet outil selon la sélection suivante:

(10) Traits conceptuels

Éléments de nomination

1. /RÈGLE/ (morphologie)

règle

2. /COULISSER/ (mode de manipulation)

3. /POUR INGÉNIEURS/ (UTILISATEUR-TYPE)

4. /CALCULER/ (fonction)

calculer

5. /LOGARITHMIQUE/ (principe théorique)

Le fait que la structure conceptuelle privilégie un certain nombre d'éléments pertinents est le propre de la stylisation conceptuelle, laquelle, sans exclure les autres traits, sélectionne les traits qui sont de nature à donner au signe linguistique une matière de contenu capable d'assurer à la fois la précision et la malléabilité de l'unité lexicale (cf. les notions de flou, approximation et non-dit chez Martin 1987: 165176).

Cet exemple a fait l'objet d'une dénomination directe dont les éléments de nomination désignent des traits conceptuels (cf. des exemples du type: «longuevue», «jeune fille», "sans mot dire», "reste à savoir si », etc.), mais il arrive souvent que la dénomination soit oblique, c'est-à-dire que ses éléments de nomination ne reprennent pas les traits conceptuels parce qu'ils sont empruntés à d'autres domaines. Dans l'exemple "passer du coq à l'âne», il est clair que «coq" et "âne » renvoient à des traits conceptuels autres que leur référent de base.

Le passage du concept au signe linguistique se fait donc de plusieurs manières mais, ce qui est certain, c'est que la description de ces mécanismes n'est possible que lorsqu'il s'agit d'unités construites, surtout les SF. 
2.4. Ce qui se passe au niveau du signe linguistique dépend en grande partie, comme nous venons de le mentionner, des moyens mis en ouvre par chaque système pour réaliser les dénominations, des spécificités morphologiques, des transferts de domaines, bref de l'économie générale de chaque langue.

Ainsi les SF, en tant que signes polylexicaux, fournissent-elles le moyen de vérifier comment le contenu conceptuel donne lieu dans le cadre d'une séquence à une synthèse sémantique par laquelle prend forme le signifié global (cf. Mejri 1997). Nous nous contentons dans ce cadre d'attirer l'attention sur la «fonction mémorielle» du lexique, étroitement liée à la dénomination oblique, illustrée par certains exemples (cf. Mejri 1998: 103-112) et de montrer comment le figement participe à la structuration du lexique.

2.4.1. Plusieurs travaux ont attiré l'attention sur la superposition dans le lexique de domaines structurés et de domaines structurants (cf. Diller 1991: 209-228; Mejri 1997).

Diller (1991: 225) a montré comment les domaines de la nourriture et de la vision «structurent [en français] partiellement les domaines de l'action verbale et de l'action mentale»: "pensées amères», "paroles aigres-douces», «mots crus», «discussion de mauvais goût», «point de vue», "vue d'esprit», «courte vue», "vue d'ensemble », etc.

De notre côté, nous avons montré comment les dénominations du corps et celles des animaux servent de moyen de dénomination du monde végétal ou d'un ensemble de comportements (Mejri 1997a: 312-325 et 391): "dent-de-cheval» (variété de topaze), « œil-de-perdrix» (variété de silex), « oreille-de-Judas» (champignon noir), «tête-de-loup» (brosse), «langue-de-vache» (enclume), «souris d'hôtel» (cambrioleuse), «vache à lait», etc.

Cette fonction mémorielle qui trahit «notre saisie symbolique du monde dans un grand nombre de domaines» (Diller 1991:210), moyennant le recours aux mécanismes tropiques, fait l'objet d'une attention particulière des linguistes (cf. les deux volumes des actes du colloque La mémoire des mots, Tunis, 1998), que cela se traduise en termes de domaines, de notions, de classes d'objets ou de structuration conceptuelle (cf. entre autres Prandi 1998a).

2.4.2. Un autre aspect, non moins important, concerne ce que nous pourrions appeler la structuration horizontale du lexique qui trouve son expression entre autres dans les rapports de synonymie établis entre unités simples du lexique et SF, phénomène permettant de mettre en lumière la conceptualisation langagière (cf. Mejri 1998c) et de montrer comment le même contenu trouve dans la SF une structure stratifiée référentiellement, conceptuellement, sémantiquement et morphologiquement pour bénéficier d'une expressivité meilleure; une telle expressivité étant le fruit de la forte prégnance assurée par la nature des rapports sémiotiques établis entre le signe polylexical et sa référence (pour les locutions familières, cf. Petit 1998). Cela concerne tous les lectes collatéraux ou les niveaux et registres de langue. Le dictionnaire de l'argot en fournit un très grand nombre d'exemples dont nous citons ceux de s'enfuir (38 séquences en tout):

(11) chier du poivre; faire l'adja; mettre les adjas; se tirer des adjas; faire natchave; faire patatrot; jouer des fourchettes; jouer des quilles; jouer un air; jouer, se tirer 
des flûtes; les agiter ; mettre les bouts, les flubes; partir comme un lavement ; prendre la tangente; ramasser, s'attacher un bidon; se déguiser en cerf, en courant d'air; se donner, prendre de l'air, changer d'air; se faire l'escarette [...]

Ainsi deux types de structuration du lexique se trouvent-ils combinés dans les SF : la première se fait par l'utilisation du lexique de certains domaines pour dénommer les entités d'autres domaines, l'autre par les équivalences établies entre signes unilexicaux et signes polylexicaux, ces derniers étant dans le cas du langage courant des désignations discursives générées par la recherche de l'autrement-dit et lexicalisées avec le temps.

3. Toutes les opérations mentionnées ont un impact certain sur le fonctionnement de ces séquences dénominatives dans le discours. Nous avons vu que les SF sont des unités où se superposent plusieurs références, concepts et signifiés; il s'ensuit qu'elles font l'objet d'emplois particuliers dans le discours.

Notons d'abord que les multiples superpositions qui distinguent les SF des autres unités du lexique réduisent énormément le caractère arbitraire du signe, ce qui autorise à y voir des unités où la valeur symbolique jouerait un rôle plus important que dans les unités simples. C'est peut-être la raison pour laquelle on y a souvent recours dans les positions les plus sensibles des textes comme le titre, le début ou la fin (cf. surtout les textes journalistiques).

3.1. S'agissant des titres, nous rappelons ceux des romans d'Hervé Bazin - La tête contre les murs, L'huile sur le feu, Un feu dévore un autre feu -, de Paule Constant Confidence pour confidence, etc. Les journalistes en usent souvent. Jugez-en par ces titres glanés dans le Canard enchaîné du 16 septembre 1998: "le mot de la fin", «Chirac met le feu», «la langue de bois de justice», «mariage sans papier», « urbi et morbide». Dans le même numéro, l'éditorial s'achève par «Autre temps, autres mœurs, même histoire!» :

(12) MONICA en VF: Cela ne se passait pas près du bureau ovale de la Maison-Blanche à Washington, entre 1996 et 1997, mais dans un urinoir des Champs-Élysées à Paris, en 1876. Les protagonistes n'étaient ni président des États-Unis ni fonctionnaire stagiaire, mais avocat de renom et bijoutier. Le premier, Eugène Lebègue, comte de Germiny, avait été surpris par la maréchaussée en posture «non convenable» avec le second, ce qui lui avait valu 2 mois de prison ferme, les honneurs de toute la presse et cette compassion de Gustave Flaubert dans une lettre adressée à Edmond Goncourt le 31 décembre 1876 [Flaubert et Goncourt, Correspondance, Paris, Flammarion, 1998]:

J'ai réellement souffert, dans ces derniers temps, de n'avoir personne à qui parler de l'affaire Germiny! Pauvre bougre, il finit par m'attendrir! - Et je trouve que la France lui doit une récompense nationale. Car enfin, il l'amuse, et tout amuseur est un bienfaiteur. La branlade d'un monsieur dans [un urinoir] a occupé la capitale $d u$ monde civilisé pendant quinze jours! Les plus belles ouvres d'art, les plus grandes découvertes scientifiques n'ont pas causé tant d'émotion quand elles ont paru! C'est beau! Ainsi la décharge de ce Bourgeois enfonce la question d'Orient! Sa manustubration [sic] avec le bijoutier (une perle!) a plus d'ampleur que la conférence des diplomates à Constantinople. La peau de ses testicules coiffe tous les Français! Nous nous égarons dans ses poils! [...]

Autre temps, autres mœurs, même histoire! 
Indépendamment de la dimension ludique propre au journal satirique, nous pensons que les unités polylexicales, y compris les proverbes, surtout lorsqu'elles sont le fruit d'une dénomination oblique, tout en signifiant, donnent à voir. Leur dualité leur offre le moyen d'assurer une fonction en quelque sorte «emblématique» qui fait que la présence de ce type d'unité condense les divers contenus dans des titres ou des formules qui annoncent ou reprennent ce qui est dilué dans le discours.

3.2. Le fonctionnement des SF dans le discours est révélateur d'emplois particuliers rattachables à la fonction que nous venons de mentionner dont le rôle structurant du discours est indéniable. Nous mentionnons en plus de cette dimension leur fonctionnement anaphorique. Observons les exemples suivants:

(13) [Son père] disait ce qui peut approximativement se traduire en français par «avoir de la bouteille», ce qui veut dire être bien chambré, bon à déguster. Elle s'était offert un «bon cru». (Constant 1998: 144).

(14) - Je voulais aussi m'excuser, dit-elle. Je n'aime pas me déballer comme ça mais à qui peut-on parler si ce n'est à une autre femme, avec d'autres femmes, pour se dire, confidence pour confidence, que finalement, ce qui est si lourd à porter nous le partageons. Elle avait vidé son sac dans une cuisine du Kansas.

C'est lourd le sac d'une femme qui vieillit avant que l'oubli ne l'allège. Il est rempli du poids d'une vie qui, la plus heureuse soit-elle, compte son lot de déceptions, gonflé du poids des autres vies qu'une femme porte en elle, celle de sa mère, celle d'une sœur, surtout si elle est morte, d'une amie... (Constant 1998: 225-226, c'est nous qui soulignons)

Dans ces deux passages, "avoir de la bouteille» et «vider son sac» sont génératrices d'éléments discursifs assurant une reprise implicite ou explicite de ces expressions. Vu leur nature métaphorique, nous parlerons d'anaphore métaphorique implicite ou explicite.

L'anaphore implicite n'est pas propre aux SF puisqu'elle repose sur la reprise d'un segment lexical par un autre segment lexical inféré par la structure conceptuelle de la dénomination du premier segment: dans (13), «bon cru» est inféré par la structure conceptuelle d' «avoir de la bouteille» et par la figuration qu'elle comporte (dans le contexte, "le bon cru» renvoie à un homme).

Ce qui n'est pas le cas de l'anaphore explicite qui engage au moins l'un des constituants de la SF: en (14), l'élément nominal de la séquence verbale, «le sac», assure l'enchaînement dans le cadre de la même métaphore et sert de support à toutes les prédications qui suivent l'emploi de la SF («lourd», «rempli», «gonflé», etc.). Ce type de reprise n'est possible qu'avec des unités polylexicales parce que leur caractère pluriel le leur permet et la dualité d'emploi des constituants le favorise.

3.3. À ce genre de construction, on peut rattacher aussi les emplois ludiques des SF, emplois favorisés par la fixité du signifiant polylexical, la sur-référenciation, la conceptualisation langagière et l'oblicité de ce genre de dénomination (pour les détails de l'analyse, cf. Mejri 1996).

En guise de conclusion, nous pouvons dire que le figement est un processus systémique dont le produit est un type de dénomination dont l'étude détaillée offre la possibilité de revisiter des questions linguistiques jugées résolues. L'oblicité de ces dénominations, la stratification de leurs structures et la polylexicalité de leurs signi- 
fiants sont autant de facteurs qui favorisent l'étude de certains concepts fondamentaux de la linguistique moderne comme la référence, la conceptualisation, l'arbitraire du signe, etc.

Dans le cadre de ce texte, nous n'avons fait qu'effleurer certaines questions fondamentales. Il reste à expliquer plusieurs phénomènes liés à la dénomination polylexicale dont nous retenons particulièrement:

- le rôle joué par la médiation du langage dans la formation du concept et son impact sur la valeur symbolique de ces signes polylexicaux;

- la difficulté à démêler données encyclopédiques et traits conceptuels;

- la manière dont la langue se structure, syntaxiquement et lexicalement, en reprenant les unités du lexique disponibles dans la communication courante;

- la dynamique autonymique des SF telle qu'elle s'exprime dans les emplois discursifs (cf. Driss Chraïbi et Raymond Queneau par exemple);

- l'établissement d'une carte des renvois opérés par le figement entre les différents domaines.

Bref autant de questions qui demeurent sans réponse!

\section{RÉFÉRENCES}

Antoine, G. et R. Martin, dir. (1995): Histoire de la langue française, 1914-1945, Paris, CNRS.

Baccouche, T., A. Clas et S. Mejri, dir. (1998): La mémoire des mots. Actes du Colloque de Tunis 1998, vol. 2, numéro spécial de la Revue tunisienne des sciences sociales, 117.

BACCOUChe, T. et S. MejRI, dir. (1998): L’information grammaticale, numéro spécial «Tunisie».

Baccouche, T., S. Mejri et M. Ghariani-Baccouche (1998): «Du sacré au profane: parcours d'une lexicalisation ", Le figement lexical. Actes de la $1^{r e}$ RLM, Tunis, septembre 1998, Tunis, CERES, p. 121-131.

BéjoInt, H., P. Thoiron et al. (1996): Les dictionnaires bilingues, Louvain-la-Neuve, Duculot et AUPELF-UREF.

Boisson, C. et P. Thoiron (1997): Autour de la dénomination, Lille, Presses universitaires de Lille.

Bosredon, B. (1998): «Les signalétiques de nomination ou quand le discours se fige», Le figement lexical. Actes de la $1^{r e}$ RLM, Tunis, septembre 1998, Tunis, CERES, p. 209-218.

Clas, A. et G. Gross (1998) : «Classes de figement des locutions verbales», Le figement lexical. Actes de la $1^{\text {re }}$ RLM, Tunis, septembre 1998, Tunis, CERES, p. 11-19.

Clas, A., S. Mejri, T. Baccouche (1997): La mémoire des mots. Actes des Ve Journées scientifiques du Réseau LTT, Tunis, septembre 1997, Tunis, AUPELF-UREF.

Colin, J.-P. et al. (1990): Dictionnaire de l'argot, Paris, Larousse.

Constant, P. (1998): Confidence pour confidence, Paris, Gallimard.

Соттеz, H. (1987): «Sur quelques problèmes de la dénomination», Cahiers de lexicologie, 50, p. $165-176$.

Darot, M. (1998): «Verlanisation et figement lexical», Le figement lexical. Actes de la $1^{\text {re }}$ RLM, Tunis, septembre 1998, Tunis, CERES, p. 321-328.

Diller, A.-M. (1991) : «Cohérence métaphorique, action verbale et action mentale», Communications, 53, p. 209-228.

Foucault, B. de (1988): Les structures linguistiques de la genèse des jeux de mots, Berne, Peter Lang, 1988.

Ghariani-Baccouche, M. (1998) : «Formules de salutation, d'une langue à l'autre. Classement et enseignement», Le figement lexical. Actes de la $1^{r e}$ RLM, Tunis, septembre 1998, Tunis, CERES, p. 329-342.

GrÉCIANO, G. (1983): Signification et dénotation en allemand. La sémantique des expressions idiomatiques, Metz, Université de Metz. 
Gross, G. (1988): «Degré de figement des noms composés, Langages, 90, p. 57-72.

- (1994): «Classes d'objets et description des verbes", Langue française, 115, p. 15-30.

- (1996): Les expressions figées, noms composés et autres locutions, Paris, Ophrys.

Gross, M. (1982) : «Une classification des phrases "figées" du français», Revue québécoise de linguistique, 11-2, p. 151-185.

Kleiber, G. (1984): «Dénomination et relations dénominatives», Langages, 76, p. 77-94.

— (1995): «Polysémie, transfert de sens et métonymie intégrée», Folia linguistica, 29-2, p. 105132.

— (1996): «Noms propres et noms communs: un problème de dénomination », Meta, 41-4, p. 567-598.

- (1998): "Les proverbes antinomiques: une grosse pierre "logique" dans le jardin toujours universel des proverbes", Le figement lexical. Actes de la $1^{\text {re }}$ RLM, Tunis, septembre 1998, Tunis, CERES, p. 51-75.

Kocourek, R. (1982): La langue française de la technique et de la science, Wiesbaden, O. Brandstetter Verlag.

Kojet El Khil, N. (1998): «Les lexies complexes à base prendre: principes de classement.», L'information grammaticale, numéro spécial «Tunisie», mai 1998, p. 23-26.

Langages (1984), 76 (numéro spécial «La dénomination»).

Le Pesant, D. et M. Mathieu-Colas (1998): «Les classes d'objets», Langages, 131.

Lobatchev, B. (1994): «Entre le motivé et l'arbitraire: l'autrement-dit», Cahiers de lexicologie, 65-2, p. 123-150.

— (1996): «Principe de la traduction - principe du langage: l'autrement-dit», Meta, 40-4, p. 707-713.

MakkaI, A. (1972): Idiom structure in English, The Hague and Paris, Mouton.

Martin, R. (1992): Pour une logique du sens, $2^{2}$ éd., Paris, Presses universitaires de France.

MejRi, S. (1994): «Séquences figées et expression de l'intensité. Essai de description sémantique», Cahiers de lexicologie, 65-2, p. 111-122.

- (1995): La néologie lexicale, La Manouba, Publications de la Faculté des lettres.

- (1997a): Le figement lexical. Descriptions linguistiques et structuration sémantique, La Manouba, Publications de la Faculté des lettres.

— (1997b): "Binarisme, dualité et séquences figées", Les formes du sens. Mélanges Robert Martin, Paris et Gembloux, Duculot, p. 249-256.

— (1997c): «Défigement et jeux de mots», Études linguistiques, vol. 3, Tunis, p. 75-92.

— (1998a): «Du figement lexical: continuité référentielle et saillance linguistique», Scolia, 11, p. 169-179.

— (1998b): «Le figement et la linéarité du signe linguistique», L'information grammaticale, numéro spécial «Tunisie», mai 1998, p. 17-21.

— (1998c) : «La conceptualisation dans les séquences figées », L’information grammaticale, numéro spécial «Tunisie», mai 1998, p. 41-48.

— (1998d): «La mémoire des séquences figées: une troisième articulation, ou la réhabilitation du culturel dans le linguistique? ", La mémoire des mots. Actes du Colloque de Tunis, septembre 1998, Tunis, AUPELF-UREF, coll. «Actualité scientifique», p. 3-11.

— (1998e): «Structuration sémantique et variation des séquences figées", Le figement lexical. Actes de la $1^{\text {re }}$ RLM, Tunis, septembre 1998, Tunis, CERES, p. 103-112.

- (1999): «Unité lexicale et polylexicalité», Linx, 40, Université Paris x-Nanterre, p. 79-96.

Meta, 41-4, décembre 1996, numéro spécial «La dénomination».

Mejri, S., G. Gross, A. Clas et T. Baccouche, dir. (1998) Le figement lexical. Actes de la $1^{\text {re }} R L M$, Tunis, septembre 1998, Tunis, CERES.

Mortureux, M.-F. (1995) : «Les techniques dans la vie quotidienne», Histoire de la langue française, 1914-1945, Paris, CNRS, p. 445-461.

Petit, G. (1998): «Remarques sur la structuration sémiotique des locutions familières », Le figement lexical. Actes de la $1^{\text {re }}$ RLM, Tunis, septembre 1998, Tunis, CERES, p. 145-163. 
Peтіот, G. (1998): "Figement lexical, discours politique, langue de bois», Le figement lexical. Actes de la $1^{\text {re }}$ RLM, Tunis, septembre 1998, Tunis, CERES, p. 293-308.

Picoche, J. (1986): Structures sémantiques du lexique français, Paris, Nathan.

Prandi, M. (1992): Grammaire philosophique des tropes, Paris, Éditions de Minuit.

- (1998): "Les motivations conceptuelles du figement", Le figement lexical. Actes de la $1^{\text {re }}$ RLM, Tunis, septembre 1998, Tunis, CERES, p. 87-101.

— (1998): «Contraintes conceptuelles sur la distribution: réflexions sur la notion de classes d'objets ", Langages, 31, p. 34-44.

Rey, A. et S. Chantreau (1989): Dictionnaire des expressions et locutions, Paris, Le Robert, coll. «Les usuels du Robert».

Vandeloise, C. (1991): «Autonomie du langage et cognition», Communications, 53, p. 209-228. 